\title{
Leptina, Elo Adicional na Fisiopatologia da Pré-eclâmpsia?
}

\author{
Leptin, Additional Link to the Pathophysiology of Preeclampsia?
}

Angela Vargas Borges Duarte, Sérgio Girão Barroso, Emílio Antônio Francischetti, Virgínia Genelhu de Abreu

\begin{abstract}
RESUM0
Objetivo: avaliar o comportamento dos niveis séricos de leptina durante a evolução da gestação de adolescentes primigestas normotensas e pré-eclâmpticas.

Métodos: estudo prospectivo e longitudinal, realizado em 15 pacientes normotensas e 5 préeclâmpticas. Os niveis séricos de leptina $(\mathrm{ng} / \mathrm{mL}$ ) foram determinados por radioimunoensaio e a pressão arterial foi aferida pelo DINAMAP 1846. Foram feitas avaliações em dois periodos gestacionais distintos: entre a $21^{\underline{a}}$ e a $30^{a}$ semana e entre a $31^{a}$ e a $40^{a}$ semana. Utilizou-se a razão leptina/indice de massa corporal (IMC) para corrigir a variação do IMC ao longo da gravidez. Considerou-se como pré-eclâmpticas as gestantes com pressão arterial $\geq 140 / 90$ $\mathrm{mmHg}$, proteinúria $>300 \mathrm{mg} / 24 \mathrm{~h}$ e espasmo arteriolar pelo exame de fundo de olho.

Resultados: quando se comparou o periodo entre a $21^{\underline{a}}$ e a $30^{a}$ semana com o periodo entre

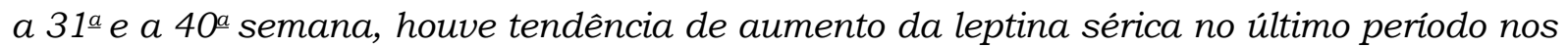
dois grupos estudados, mais acentuadamente nas pré-eclâmpticas que nas grávidas

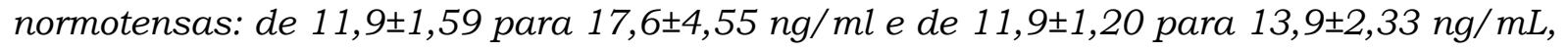
respectivamente. O IMC de gestantes normotensas não se alterou nos dois periodos analisados: $24,9 \pm 1,5 \mathrm{~kg} / \mathrm{m}^{2}$ vs $25,2 \pm 1,0 \mathrm{~kg} / \mathrm{m}^{2}$. Entretanto, o IMC aumentou significativamente nas pré-eclâmpticas no final da gravidez: $21,5 \pm 0,8$ vs $27,4 \pm 1,7 \mathrm{~kg} / \mathrm{m}^{2}, p<0,05$. Nas préeclâmpticas a razão leptina/IMC elevou-se significativamente no final da gestação: de 0,56士 0,06 (da $21^{\underline{a}}$ à $30^{a}$ semana) vs $0,70 \pm 0,15$ (entre 31 e 40 semanas), $p<0,05$. Os valores da

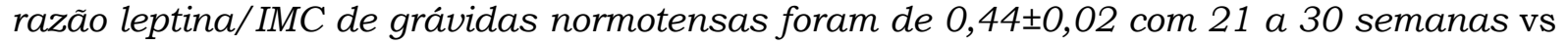
$0,41 \pm 0,04$ com 31 a 40 semanas. Nas gestantes normais a leptina se correlacionou direta e significativamente com o IMC tanto entre a $21^{\underline{a}}$ e a $30^{\underline{a}}$ semana $(r=0,7, p<0,004)$, como entre

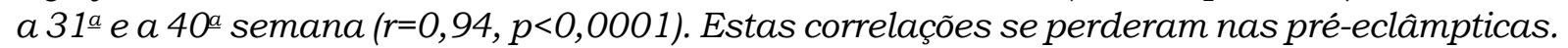
Conclusão: o aumento das concentrações séricas de leptina e da relação leptina/IMC nas pré-eclampticas em relação às normotensas, nos dois periodos gestacionais, sugere resistência à ação da leptina na pré-eclâmpsia.
\end{abstract}

PALAVRAS-CHAVE: Complicações da gravidez. Leptina. Hipertensão arterial. Índice de massa corporal.

\section{Introdução}

A leptina, um polipeptídeo com 167 aminoácidos, foi originalmente descrita como hormônio das células adiposas ${ }^{1}$. Caracterizada como produto do gene ob, modula o apetite e controla os depósitos de gordura em indivíduos

Laboratório de Fisiopatologia Clinica e Experimental CLINEX, Universidade do Estado do Rio de Janeiro - UERJ Correspondência:

Virginia Genelhu de Abreu

Rua Paulo César de Andrade, 106/ 602 - Laranjeiras

22221-090 - Rio de Janeiro - RJ eutróficos ${ }^{1}$. A leptina circulante liga-se a receptores do plexo coróide e atua no cérebro sobre receptores hipotalâmicos. Esses receptores estabelecem o elo de ligação entre leptina e sistema nervoso simpático, leptina e gonadotrofinas e leptina e mediadores do apetite e termogênese (neuropeptídeo y, melanocortinas e outros) ${ }^{2}$. Reconhece-se, atualmente, que a leptina, além de ser importante mediador dos depósitos somáticos de energia, atua no controle da fertilidade e crescimento. Mutações no gene ob são responsáveis pela obesidade extrema e infertilidade observadas em camundongos ob/ob ${ }^{1}$. 
Em humanos são raros os indivíduos com obesidade extrema devido à expressão de gene mutante, não funcionante da leptina ${ }^{2}$. Obesos humanos costumam ter níveis elevados de leptina circulante caracterizando um "estado de resistência" ao polipeptídeo ${ }^{2}$.

As concentrações séricas de leptina são maiores na mulher grávida do que na mulher não grávida ${ }^{3}$. Aumentos substanciais dos niveis de leptina ocorrem no início da gravidez, antes de qualquer elevação significativa do peso corporal, sugerindo que outros fatores além da adiposidade participariam do controle dos niveis séricos de leptina ${ }^{4}$. As concentrações de leptina atingem um pico no segundo trimestre da gestação, persistindo elevadas até o parto. O papel fisiológico proposto para a leptina durante a gravidez ainda não está plenamente esclarecido, sugerindo-se seu envolvimento na regulação do crescimento e desenvolvimento fetal. Ainda se desconhece a origem da leptina circulante neste período. Recentemente, descreveu-se a expressão de leptina e de seus receptores na placenta ${ }^{5}$. Discute-se se a leptina de origem placentária atuaria diretamente no hipotálamo, independentemente da leptina dos adipócitos ${ }^{6}$. Em situações de disfunção placentária existe maior produção de leptina ${ }^{7}$. A hipóxia placentária se associa a níveis elevados de leptina? ${ }^{7}$.

Tendo em vista que os trabalhos mais consistentes sobre pré-eclâmpsia foram realizados em primigrávidas $^{8}$ e que o diagnóstico de préeclâmpsia é freqüentemente equivocado em multiparas, resolveu-se conduzir o presente estudo com o objetivo de avaliar a participação da leptina durante a evolução da gestação em grupo de alto risco -adolescentes primigestas-, tendose estudado não só o comportamento dos níveis séricos do hormônio como a relação leptina/indice de massa corporal (IMC), corrigindo-se para a variação do IMC durante a progressão da gravidez.

\section{Pacientes e Métodos}

Trata-se de um estudo prospectivo e longitudinal realizado em gestantes adolescentes primigestas. As pacientes foram classificadas segundo a idade gestacional em 4 grupos: Grupo 1, com gravidez entre a $21^{\text {a }}$ e a $30^{a}$ semanas, constituído de 14 normotensas; Grupo 2, entre a 21 $1^{\text {a }}$ e a 30 a semanas, com 5 gestantes com doença hipertensiva; Grupo 3 (31 à 40 ${ }^{\mathrm{a}}$ semana), com 12 normotensas, e Grupo 4 (31a à 40a semana), com 5 gestantes portadoras de doença hipertensiva específica da gravidez. As gestantes classificadas como normotensas evoluíram até o final da gravidez sem hipertensão. As pacientes foram selecionadas no Serviço de Obstetrícia do Hospital Universitário Pedro Ernesto, Universidade do Estado do Rio de Janeiro, de acordo com critérios estabelecidos pelo protocolo do estudo e encaminhadas ao Laboratório de Fisiopatologia Clínica e Experimental - CLINEX, onde o estudo foi conduzido.

O presente estudo se iniciou após aprovação pelo Comitê de Ética do Hospital Universitário Pedro Ernesto e após consentimento informado das pacientes.

Foram consideradas pré-eclâmpticas as adolescentes primigestas sem antecedentes prévios de hipertensão, que desenvolveram hipertensão com proteinúria significante e edema de mãos e/ ou face, com início dos sintomas após a 20a semana de gestação ${ }^{9,10}$. Como critério de diagnóstico de hipertensão na gestação foi considerado um aumento de $30 \mathrm{mmHg}$ ou mais nos niveis de pressão arterial sistólica, ou um aumento de $15 \mathrm{mmHg}$ ou mais nos niveis de pressão arterial diastólica, ou ainda uma pressão arterial sistólica de pelo menos $140 \mathrm{mmHg}$ ou uma pressão arterial diastólica de pelo menos $90 \mathrm{mmHg}$. Estes níveis foram confirmados após período mínimo de 6 horas, estando a gestante em repouso. Considerouse proteinúria significativa aquela cujo valor foi de pelo menos $0,3 \mathrm{~g} / \mathrm{L}$ em urina de 24 horas, ou 1 $\mathrm{g} / \mathrm{L}$ ( 1 cruz ou mais pelo método semiquantitativo de fita) em amostras simples. O edema gestacional foi caracterizado por cacifo de $1 \mathrm{cruz}$ ou mais, após repouso de 12 horas no leito, ou pelo aumento de peso de $2.275 \mathrm{~g}$ em uma semana.

Foram estudadas também gestantes adolescentes, primigestas, normotensas (PA <130/85 $\mathrm{mmHg}$ ) e que não estavam utilizando qualquer medicação, exceto vitaminas e/ou suplementação oral de ferro, excluindo-se desse grupo as que apresentavam hipertensão ou antecedentes de hipertensão arterial.

A pressão arterial foi mensurada por método oscilométrico, utilizando-se equipamento DINAMAP 1846, aferindo-se a variável na posição sentada e obtendo-se a média de 3 medidas com intervalos de 5 minutos entre cada avaliação. Amostras de sangue periférico foram colhidas após jejum noturno de 12 horas, centrifugadas a 3000 rpm durante 10 minutos e armazenadas a $-20^{\circ} \mathrm{C}$ até as determinações. A leptina sérica foi estimada por radioimunoensaio utilizando-se "kit" comercial (Linco, HL-81K). Para ajustar as variações da leptina em relação ao peso utilizou-se a razão das concentrações séricas de leptina sobre o IMC, expressando-se as concentrações de leptina (ng/ $\mathrm{mL})$ por unidade de IMC $\left(\mathrm{kg} / \mathrm{m}^{2}\right)$. O IMC foi calcu- 
lado dividindo-se o peso em quilogramas pelo quadrado da altura em metros.

Os resultados foram expressos como média \pm EPM. O teste $t$ de Student foi utilizado nas análises de comparação da razão leptina /IMC entre gestantes normotensas e pre-eclâmpticas, e o de Pearson nas análises de correlação dos níveis de leptina com o IMC.

\section{Resultados}

Não houve diferença significativa quanto à idade entre normotensas e pré-eclâmpticas.

Os niveis de leptina aumentaram nas normotensas e nas pré-eclâmpticas no período entre a $21^{\text {a }}$ e a $30^{\mathrm{a}}$ semana quando comparados ao período da $31^{\text {a }}$ à $40^{a}$ semana $(11,9 \pm 1,2 \mathrm{ng} / \mathrm{mL}$ para $13,9 \pm 2,3 \mathrm{ng} / \mathrm{mL}$, e $11,3 \pm 1,6$ para $17,6 \pm 4,5$, respectivamente). Essas diferenças não foram significativas, não havendo também diferença significativa entre normotensas e pré-eclâmpticas.

O IMC de gestantes normotensas não se al-

a) Gestantes normais: $21^{\mathrm{a}}$ a $30^{\mathrm{a}}$ semana

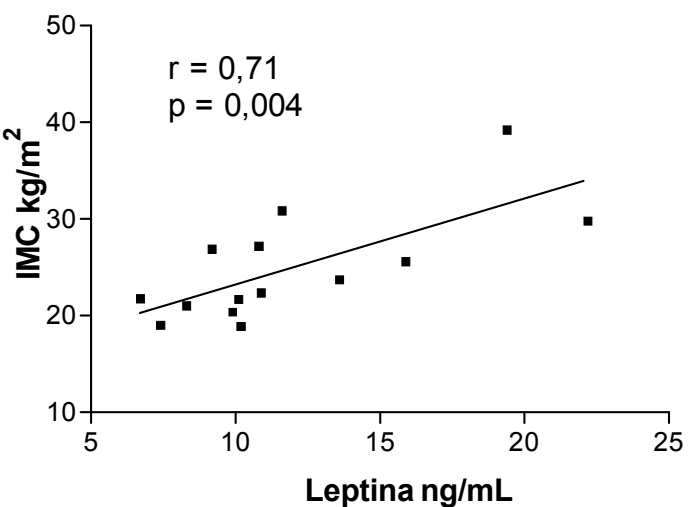

terou nos dois períodos gestacionais analisados $\left(24,9 \pm 1,5 \mathrm{~kg} / \mathrm{m}^{2}\right.$ us $\left.25,2 \pm 1,0 \mathrm{~kg} / \mathrm{m}^{2}\right)$. Entretanto, nas pré-eclampticas os valores do IMC aumentaram significativamente do período entre a $21^{\text {a }}$ e a $30^{a}$ semana para o período com mais de 31 semanas $\left(21,5 \pm 0,8 \mathrm{~kg} / \mathrm{m}^{2}\right.$ vs $27,4 \pm 1,7 \mathrm{~kg} / \mathrm{m}^{2}$, respectivamente, $p=0,03$ ).

Nas gestantes normais a leptina se correlacionou direta e significativamente com o IMC, no período entre a $21^{\underline{a}}$ e a $30^{\text {a }}$ semana $(r=$ $0,7, \mathrm{p}<0.004)$ e no período da $31^{\mathrm{a}}$ à $40^{\mathrm{a}}$ semana de gestação $(r=0,94 ; p<0,0001)$ (Figura 1a e b). Estas correlações se perderam nas pré-eclâmpticas, tanto nas que estavam entre a $21^{\text {a }}$ e a $30^{\text {a }}$ semana de gravidez $(\mathrm{r}=0,8 ; \mathrm{p}=0,09)$, como naquelas entre a $31^{\mathrm{a}}$ e a $40^{a}$ semana $(r=0,7 ; p=N S)$. Embora os niveis de leptina não tenham sido significativamente diferentes entre normotensas e pré-eclâmpticas, a relação leptina/IMC foi significativamente maior na gravidez que cursou com pré-eclâmpsia, tanto no grupo com 21 a 30 semanas quanto naquele com mais de 31 semanas, quando se comparou com o grupo de grávidas normotensas (Figura 2a e b).

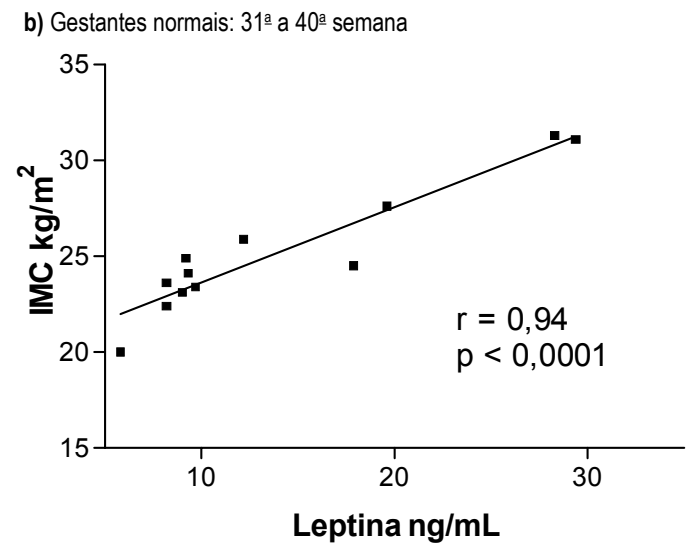

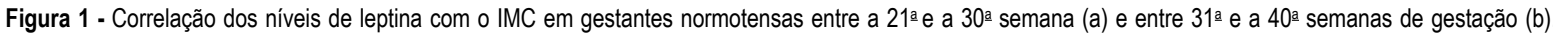
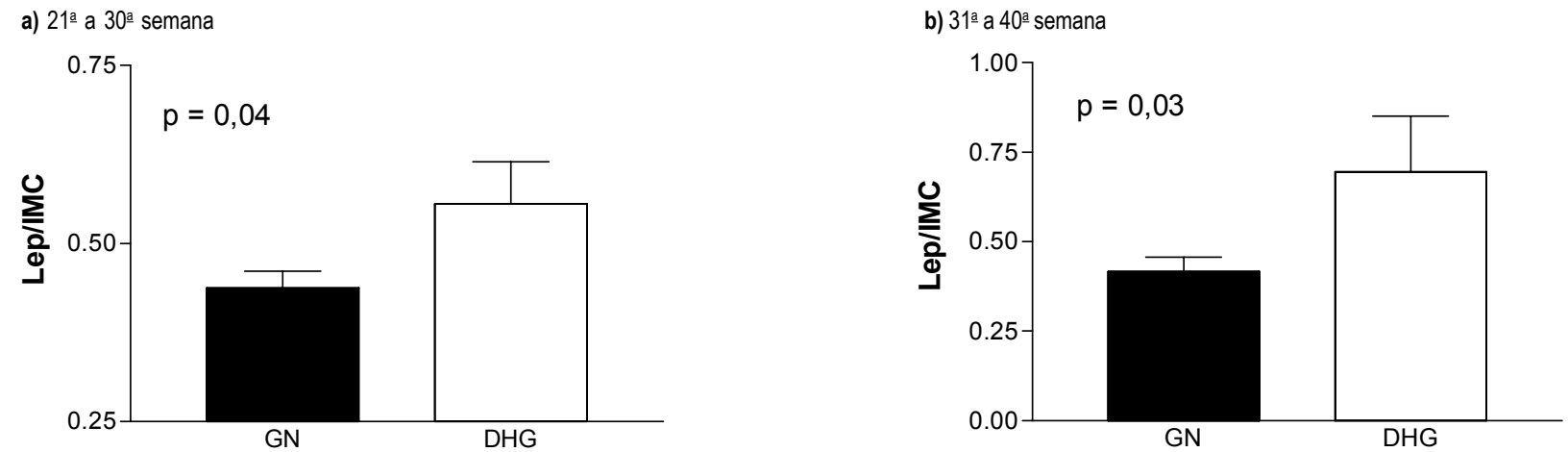

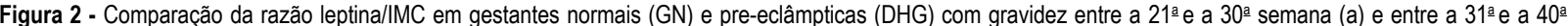
semana de gestação $(b)$. 


\section{Discussão}

A pré-eclâmpsia é uma síndrome cujas manifestações clínicas aparecem na segunda metade da gravidez e está geralmente associada a hipóxia placentária ${ }^{7}$. Embora a gestação seja um momento de importantes mudanças no peso corporal materno, os mecanismos que regulam essas alterações são pouco conhecidos. Contudo, a recente caracterização da leptina como proteína sintetizada pelo tecido adiposo e com importantes ações reguladoras de neuropeptídeos moduladores do apetite, peso, fertilidade e balanço energético ${ }^{11,12}$ significou um passo adicional para o entendimento de elos fisiológicos e fisiopatológios da gestação normal e da pré-eclâmpsia. Em 1997, descreveu-se a expressão de RNAm da leptina pela placenta, bem como de seus receptores, em roedores ${ }^{13}$. Foi também demonstrado em humanos que a leptina circulante durante a gravidez pode ter origem placentária ${ }^{14}$ e que seus niveis estão elevados no início da gestação ${ }^{4,15}$.

Os resultados de nosso estudo mostram que as concentrações séricas de leptina tendem a se elevar mais nas pré-eclampticas, no último periodo da gestação, que nas grávidas normais. Além disso, o IMC das grávidas que cursaram com préeclâmpsia aumentou significativamente quando se compara o período compreendido entre a $21^{\underline{a}} \mathrm{e}$ a $30^{a}$ semana com o período entre a $31^{\text {a }}$ e a $40^{\text {a }}$ semana, o que não ocorreu com gestantes normais. Isto poderia ser explicado pelo aumento do percentual de adiposidade, mais pronunciado no último período gestacional. Por outro lado, em grávidas normais a leptina correlacionou-se diretamento ao IMC. Esse aumento teria um papel fisiológico, uma vez que as ações da leptina seriam dirigidas para tarefas específicas da nutrição materna, com um melhor aproveitamento da energia consumida em antecipação à lactação ${ }^{15-17}$.

Nas pré-eclâmpticas a correlação leptina vs IMC se perdeu provavelmente pela contribuição do maior conteúdo de sódio corporal ao IMC, ou uma produção de leptina aumentada por outros tecidos que não o adiposo. Esta seria uma possibilidade plausivel tendo em vista o aumento desproporcional das concentrações de leptina por unidade de IMC a partir da 20aㅗ semana da gestação complicada por hipertensão, quando comparada à gravidez normal, configurando, assim, um estado de resistência à leptina nas pacientes que cursaram com pré-eclâmpsia (Figura $2 \mathrm{a}$ e b). O fato de níveis séricos de leptina atuarem na up-regulation de receptores do sistema nervoso simpático ${ }^{18,19}$ contribuindo para significativas mudanças meta- bólicas e hemodinâmicas, estaria em consonância com a hipótese de um estado simpaticotônico na pré-eclâmpsia ${ }^{20}$.

Contudo, o presente estudo tem limitações. Primeiro, o pequeno número de pacientes com préeclâmpsia pode ter subestimado os resultados de leptina nas pré-eclâmpticas. Segundo, não se mediu diretamente a adiposidade corporal, não se excluindo, assim, alguns fatores de confusão a respeito dessa variável quando expressa pelo IMC e, em terceiro lugar, a atividade simpática não foi avaliada.

Em conclusão, nossos resultados mostram que primigestas adolescentes que cursam com préeclâmpsia apresentam resistência às ações da leptina, o que pode ser um elo adicional à etiopatogenia da doença. São resultados preliminares e estudos mais detalhados, com maior número de pacientes, precisam ser realizados para testar apropriadamente esta hipótese.

\section{SUMMARY}

Purpose: to study in primigravid adolescents the behavior of serum leptin levels during the evolution of normal pregnancy, comparing the results with those obtained from preeclamptics.

Methods: prospective, longitudinal study conducted in 15 normotensive pregnant adolescents and 5 preeclamptic adolescents. Serum leptin levels $(\mathrm{ng} / \mathrm{mL})$ were determined by radioimmunoassay. Blood pressure was measured by the oscilometric method by using DINAMAP 1846. Patients were evaluated in two different gestational periods: between the 21 st and 30th week and between the 31st and 40th week. The ratio leptin/body mass index (BMI) was used to correct changes observed in BMI throughout gestation. Preeclamptic pregnant patients were diagnosed when the blood pressure was $\geq 140 / 90 \mathrm{mmHg}$, proteinuria $>300 \mathrm{mg} /$ $24 \mathrm{~h}$ and when arteriolar spasm was present in the optic fundi.

Results: there was a trend towards an elevation of serum leptin at the end of pregnancy in both groups although more pronounced in preeclamptic patients. In pregnant normotensive patients serum leptin increased from $11.9 \pm 1.20$ (21st to 30 th week) to $13.9 \pm 2.23 \mathrm{ng} / \mathrm{mL}$ (31 st to 40 th week), and in preeclamptic from $11.9 \pm 1.20$ to $17.6 \pm 4.565 \mathrm{ng} / \mathrm{mL}$. In preeclamptic patients the BMI increased significantly in the period from the 21st to 30th week when compared to the period between the 31 st and 40 th week: $21.5 \pm 0.8$ vs $27.4 \pm 1.7$ $\mathrm{kg} / \mathrm{m}^{2}, p<0.05$. In normotensive these values were maintained stable: $24.9 \pm 1.5$ vs $25.1 \pm 1.00 \mathrm{~kg} / \mathrm{m}^{2}$. At the end of gestation the ratio leptin/BMI was significantly higher in preeclamptics: $0.56 \pm 0.06$ (21st to 30 th week) vs $0.70 \pm 0.15$, $p<0.05$. The values of the ratio leptin/BMI in normotensive pregnants varied from $0.44 \pm 0.02$ between the 21 st and 30 th week to $0.41 \pm 0.04$ between the 31st to 40th week. In normal 
pregnants there was a direct and significant correlation between the levels of leptin and BMI in both periods of pregnancy: $r=0.7, p<0.004$ (31st to 40th) vs $r=0.94$, $p<0.0001$ (31st to 40th week). These correlations were lost in preeclamptic patients in both gestational periods.

Conclusion: the higher concentrations of leptin and leptin/ BMI ratio observed when preeclamptics were compared with normotensive patients, in both gestational periods, suggest a leptin resistance pattern in preeclampsia.

KEY WORDS: Complications of pregnancy. Hypertension. Leptin. Body mass index.

\section{Referências}

1. Zhang Y, Proenca R, Maffei M, Barone M, Leopold L, Friedman JM. Positional cloning of the mouse obese gene and its human homologue. Nature 1994; 372:425-32.

2. Schwartz MW, Woods SC, Porte D Jr, Seeley RJ, Baskin DG. Central nervous system control of food intake. Nature 2000; 404:661-71.

3. Henson MC, Castracane VD. Leptin in pregnancy. Biol Reprod 2000; 63:1219-28.

4. Highman TJ, Friedman JE, Huston LP, Wong WW, Catalano PM. Longitudinal changes in maternal serum leptin concentration, body composition, and resting metabolic rate in pregnancy. Am J Obstet Gynecol 1998; 178:1010-5.

5. Mantzoros CS. The role of leptin in human obesity and disease: a review of current evidence. Ann Intern Med 1999; 130:671-80.

6. Bi S, Gavrilova O, Gong DW, Mason MM, Reitman M. Identification of a placental enhancer for the human leptin gene. J Biol Chem 1997; 272:305838.

7. Mise H, Sagawa N, Matsumoto T, et al. Augmented placental production of leptin in preeclampsia: possible involvement of placental hypoxia. J Clin Endocrinol Metab 1998; 83:3225-9.

8. Taylor DJ. The epidemiology of hypertension during pregnancy. In: Rubin PC, editor. Hypertension in Pregnancy. $1^{\text {st }}$ ed. Amsterdam: Elsevier; 1988. p.223-40.
9. Hughes EC, editor. Obstetric gynecologic terminology. Philadelphia: F.A. Davis; 1972. p.422-6.

10.Zugaib M, Kahhale S. Conceito, classificação e incidência das sindromes hipertensivas na gestação. Ginecol Obstet Bras 1985; 8:239-44.

11.Buchanan C, Mahesh V, Zamorano P, Brann D. Central nervous system effects of leptin. Trends Endocrinol Metab 1998; 9:146-50.

12.Bray GA, York DA. Leptin and Clinical Medicine: a new piece in the puzzle of obesity. J Clin Endocrinol Metab 1997; 82:2771-6.

13.Hoggard N, Hunter HL, Duncan JS, Williams LM, Trayhurn P, Mercer JG. Leptin and leptin receptor mRNA and protein expression in the murine fetus and placenta. Proc Natl Acad Sci USA 1997; 94:11073-8.

14.Masuzaki H, Ogawa H, Sagawa N, et al. Nonadipose tissue production of leptin: leptin as a novel placenta-derived hormone in humans. Nat Med 1997; 3:1029-33.

15.Schubring C, Englaro P, Siebler T, et al. Longitudinal analysis of maternal serum leptin levels during pregnancy, at birth and up to six weeks after birth: relation to body mass index, skinfolds, sex steroids and umbilical cord blood leptin levels. Horm Res 1998; 50:276-83.

16.Mukherjea R, Castonguay TW, Douglass LW, MoserVeillon P. Elevated leptin concentration in pregnancy and lactation: possible role as a modulator of substrate utilization. Life Sci 1999; 65:1183-93.

17. Chehab FF. Leptin as a regulator of adipose mass and reproduction. Trends Pharmacol Sci 2000; 21:309-14.

18.Haynes WG, Morgan DA, Walsh SA, Sivitz WI, Mark AL. Cardiovascular consequences of obesity: role of leptin. Clin Exp Pharmacol Physiol 1998; 25:65-9.

19.Haynes WG, Sivitz WI, Morgan DA, Walsh SA, Mark AL. Sympathetic and cardiorenal actions of leptin. Hypertension 1997; 30:619-23.

20.Hermida RC, Ayala DE, Mojón A, et al. Blood pressure patterns in normal pregnancy, gestational hypertension, and preeclampsia. Hypertension 2000; 36:149-58. 